\title{
A prática profissional e o cuidado de enfermagem em Saúde Mental: uma revisão
}

\section{integrativa}

\author{
Professional practice and nursing care in Mental Health: an integrative review \\ Práctica profesional y cuidados de enfermería en Salud Mental: una revisión integradora
}

\author{
Amanda dos Santos Mota \\ ORCID: https://orcid.org/0000-0003-0985-3379 \\ Universidade Federal Fluminense, Brasil \\ E-mail: amandas_mota@yahoo.com.br \\ Ana Lúcia Abrahão \\ ORCID: https://orcid.org/0000-0002-0820-4329 \\ Universidade Federal Fluminense, Brasil \\ E-mail: anaabrahao@id.uff.br \\ Uirá Duarte Wisnesky \\ ORCID: https://orcid.org/0000-0002-0643-6097 \\ Universidade de Alberta, Canadá \\ E-mail: uira.wisnesky@ualberta.ca \\ Taís Veronica Cardoso Vernaglia \\ ORCID: https://orcid.org/0000-0003-3391-7301 \\ Universidade do Estado do Rio de Janeiro, Brasil \\ E-mail: tais.vernaglia@unirio.br \\ Carlos Magno Carvalho da Silva \\ ORCID: https://orcid.org/0000-0002-8655-2636 \\ Universidade do Estado do Rio de Janeiro, Brasil \\ E-mail: cmagnocarvalho1@gmail.com \\ Rafael Vieira Braga da Silva \\ ORCID: https://orcid.org/0000-0003-4797-8324 \\ Universidade do Estado do Rio de Janeiro, Brasil \\ E-mail: rafaelvb@uol.com.br
}

\begin{abstract}
Resumo
Objetivo: analisar as práticas de enfermagem em estudos na área psiquiátrica/de saúde mental. Métodos: O processo de revisão integrativa foi usado para conduzir esse estudo. Quatro bases de dados - LILACS, MEDLINE, SCOPUS e CINAHL - foram pesquisadas em busca de estudos elegíveis usando palavras-chave relevantes. A estratégia PICo foi empregada para a seleção dos estudos. Estudos primários publicados em português e inglês de 2010 a 2020 foram revisados seguindo critérios de inclusão pré-determinados. Os estudos que atenderam aos critérios de inclusão foram avaliados usando a recomendação de Stetler et al. (1998). Resultados: foram analisadas 15 publicações. A diversidade das práticas de enfermagem evidenciadas envolve ações de administração de medicamentos, aferição de sinais vitais, higiene, alimentação, além de ações que visavam a autoestima e autocuidado. Também foram identificados estudos com ações centradas na singularidade do usuário. Dos artigos analisados, foi possível extrair o total de 15 atividades práticas, o que demonstra de fato um leque amplo no papel compreendido dessa profissão. A assistência junto a medicação dos usuários foi a função mais citadas pelos autores. Conclusões: a execução das práticas de enfermagem em saúde mental deve ser encarada como um desafio que ultrapasse o padrão medicamentoso e que crie propostas de cuidado com foco no usuário. Esse cuidado pautado no sujeito como potência mostrou-se presente na dinâmica de trabalho dos CAPS.
\end{abstract}

Palavras-chave: Enfermagem; Saúde mental; Enfermagem psiquiátrica; Cuidados de enfermagem; Prática profissional.

\begin{abstract}
Objective: to analyze nursing practices in studies within the psychiatric/mental health area. Methods: An integrative review process was used to guide this study. Four databases - LILACS, MEDLINE, SCOPUS and CINAHL —were searched for eligible studies using relevant keywords. PICo strategy was employed for selection of studies. Primary studies published in Portuguese and English language from 2010 to 2020 were reviewed following a pre-determined inclusion criteria. Studies that met the inclusion criteria were appraised using the recommendation from Stetler at al. (1998).Results: 15 publications were analyzed. The evidence showed the diversity of nursing practices involved medication administration, measurement of vital signs, hygiene, nutrition, in addition to actions aimed at self-esteem and self-care. Studies about nursing actions focused on the uniqueness of the patient were also identified. From the analyzed articles, it was possible to extract a total of 15 practical nursing activities, which, in fact, demonstrates a wide range in the understood role of the nursing profession. Assistance to patients' medication was the most cited activity by the authors. Conclusions: the implementation of nursing practices in mental health must be seen as a challenge that goes
\end{abstract}


beyond the medication standard and creates care proposals with a focus on the patient. This patient centered care was present in the work dynamics of the CAPS.

Keywords: Nursing; Mental health; Psychiatric nursing; Nursing care; Professional practice.

\section{Resumen}

Objetivo: analizar las prácticas de enfermería en estudios dentro del área de psiquiatría / salud mental. Métodos: Se utilizó un proceso de revisión integradora para guiar este estudio. Se buscaron estudios elegibles en cuatro bases de datos - LILACS, MEDLINE, SCOPUS y CINAHL - mediante palabras clave relevantes. Se utilizó la estrategia PICo para la selección de estudios. Los estudios primarios publicados en portugués e inglés de 2010 a 2020 se revisaron siguiendo unos criterios de inclusión predeterminados. Los estudios que cumplieron con los criterios de inclusión se evaluaron utilizando la recomendación de Stetler et al. (1998) Resultados: Se analizaron 15 publicaciones. La evidencia mostró la diversidad de prácticas de enfermería que involucran la administración de medicamentos, medición de signos vitales, higiene, nutrición, además de acciones dirigidas a la autoestima y el autocuidado. También se identificaron estudios con acciones de enfermería enfocadas en la singularidad del paciente. De los artículos analizados, fue posible extraer un total de 15 actividades prácticas de enfermería, lo que, de hecho, demuestra una amplia gama en el rol entendido de la profesión de enfermería. La asistencia con la medicación de los pacientes fue la actividad más citada por los autores. Conclusiones: la implementación de las prácticas de enfermería en salud mental debe ser vista como un desafío que va más allá del estándar de medicación y genera propuestas de cuidados con enfoque en el paciente. Esta atención centrada en el paciente estuvo presente en la dinámica de trabajo del CAPS.

Palabras clave: Enfermería; Salud mental; Enfermería psiquiátrica; Cuidado de enfermera; Practica profesional.

\section{Introdução}

O cuidado psiquiátrico esteve, por um longo tempo, centrado na contenção e isolamento social dos usuários. Com o passar do tempo, espaços asilares, e mais tarde os hospitais psiquiátricos, foram criados com o intuito de ações de vigilância e manutenção básica da vida dos doentes. Durante esse período, a enfermagem não era institucionalizada como profissão. A prática centrava-se em um fazer tácito, em que as respostas iniciais eram intuitivas, com base em solução dos problemas, formando assim um corpo de conhecimento que gradualmente se desenvolveu e expandiu até para o que mais tarde, denominaríamos de ciência da Enfermagem (Mota, Silva, \& Souza, 2016).

Dessa forma, as transformações no papel do enfermeiro(a) psiquiátrico(a), ocorreram concomitante à mudança da assistência prestada nessas instituições. Observa-se que a partir dos anos 1930 até os anos 1950 na assistência psiquiátrica, houve a entrada da abordagem psicológica e social, implementada pelas novas técnicas e políticas direcionadas ao tratamento do usuário (Costa, Moraes Filho, \& Souza, 2019). É nessa atmosfera de novos conhecimentos que a enfermagem começa as primeiras incursões, para uma prática pautada na sistematização científica com enfermeiras utilizando modelos teóricos sistematizados para descrever a práxis da Enfermagem Psiquiátrica baseada no processo interpessoal.

Desse período da história até os dias atuais, tivemos o desenrolar de muitos acontecimentos, ações, estratégias, como o próprio movimento da Reforma Psiquiátrica no Brasil. A Itália possui um papel de destaque no que diz respeito a experiências inovadoras, com Franco Basaglia (Serapioni, 2019). Esta parte de experiências prévias e propõe transformações teórico-práticas na psiquiatria e na assistência em saúde mental. Houve uma mudança do paradigma, não mais a doença era o foco, e sim o sujeito-social com transtorno mental. Consequentemente, a remissão do sintoma não era mais a finalidade do cuidado, mas as necessidades de saúde propriamente humanas. Além disso, os instrumentos de intervenção (os saberes e as práticas) foram legitimados com os profissionais de saúde mental (Pan American Health Organization, 2018).

Mundialmente, a saúde mental é cada vez mais reconhecida como uma prioridade global de saúde e desenvolvimento econômico. Os transtornos mentais já representam quatro das dez principais causas de incapacidade em todo o mundo. Esse crescente ónus representa um custo enorme em termos de sofrimento humano e incapacidade pública (Pan American Health Organization, 2018). No Brasil, estimativas recentes mostraram que os transtornos depressivos e de ansiedade respondem, respectivamente, pela quinta e sexta causa de anos de vida vividos com incapacidade (Lopes, 2020). 
Diante do exposto, tem sido um desafio permanente as novas convocações trazidas a partir da Reforma Psiquiátrica no Brasil, no sentido de enfrentar os saberes instituídos historicamente que produzem a institucionalização do portador de transtorno psíquico - marcada pela violência e pelo isolamento -, na busca por práticas a favor da cidadania e da redemocratização dos espaços de convivência e tolerância. Há uma demanda pela produção de novos modos de atenção à saúde mental que requerem transformações dos saberes teóricos e práticos das profissões. Neste contexto, torna-se importante incorporar a prática de enfermagem aspectos que conjuguem cuidado com a dinâmica social do sujeito em sofrimento psíquico. Desta forma, o objetivo deste estudo foi analisar o emprego das ações práticas de enfermagem em estudos na área psiquiátrica/saúde mental.

\section{Metodologia}

Este é um estudo de revisão integrativa da literatura que tem como proposta sintetizar e avaliar criticamente as evidências relacionadas ao tema estudado de forma a fundamentar um estudo significativo. Este tipo de apreciação exige dos revisores que a análise e a síntese dos dados levantados durante a pesquisa ocorram de forma sistemática e rig orosa (Cieto, Garbuio, Camargo, \& Napoleão, 2014). Nesse sentido, optamos por seguir com a estratégia PICo para estruturar a busca nas bases de dados: P - Cuidado de enfermagem; I - Prática profissional; Co- campo da Saúde Mental. A princípio, identificou-se a questão do estudo: como se constituem as práticas e o cuidado de enfermagem em saúde mental na produção cientifica?

No processo de busca dos dados, foi realizado levantamento nas bases de dados Cumulative Index to Nursing and Allied Health Literature (CINAHL), Medical Literature Analysis and Retrieval System Online (MEDLINE), Literatura LatinoAmericana em Ciências da Saúde (LILACS) e Scopus. Empregou-se os descritores: "enfermagem psiquiátrica"; "cuidados de enfermagem"; "prática profissional"; isolados e combinados pelos operadores booleanos AND e OR. Na formulação das 'strings', foram usados os sinônimos de cada descritor no intuito de aumentar nossas chances de abrangência. Para base de dados CINAHL, o recorte temporal foi menor, dos últimos 3 anos, levando em consideração o seu foco amplo em artigos e periódicos em enfermagem, possibilitando acesso a um número maior de estudos. As referidas bases foram acessadas sem custos, através do portal de periódicos Capes.

Foram incluídos no estudo: a) artigos originais de pesquisa; b) disponibilizados nas bases de dados na íntegra; c) publicados no período de 2010 a 2020, no idioma inglês e português; d) na área de saúde sobre as práticas/e ou cuidado de enfermagem em saúde mental. Definimos como critérios de exclusão: a) trabalhos cuja abordagem da prática de enfermagem em saúde mental não foi o foco central do estudo nos resultados e conclusões; b) artigos de revisão em geral; c) artigos duplicados.

A pesquisa nas bases de dados eleitas para o estudo ocorreu entre agosto e setembro de 2020, com a aplicação de formulário avançado, com objetivo de extrair dados de forma organizada, seguindo os seguintes itens: base de dados; título do artigo, autor, revista, volume, número, página, ano de publicação, país e idioma do estudo, estratégia metodológica, objetivo do estudo, resultados e limitações do estudo.

Após a identificação do material, realizou-se a seleção dos estudos, de acordo com a estratégia PICo, e os critérios de inclusão e exclusão previamente definidos. Foi empregado o gerenciador de referências Mendeley para o armazenamento dos estudos e identificação de duplicidade. Na sequência, para conduzir a organização das informações utilizouse o checklist Preferred Reporting Items for Systematic Reviews and Meta-Analyses (PRISMA) (Figura 1). 
Figura 1 - Fluxograma PRISMA das etapas de seleção das publicações para o estudo de revisão integrativa - Niterói, RJ, Brasil, 2020 .
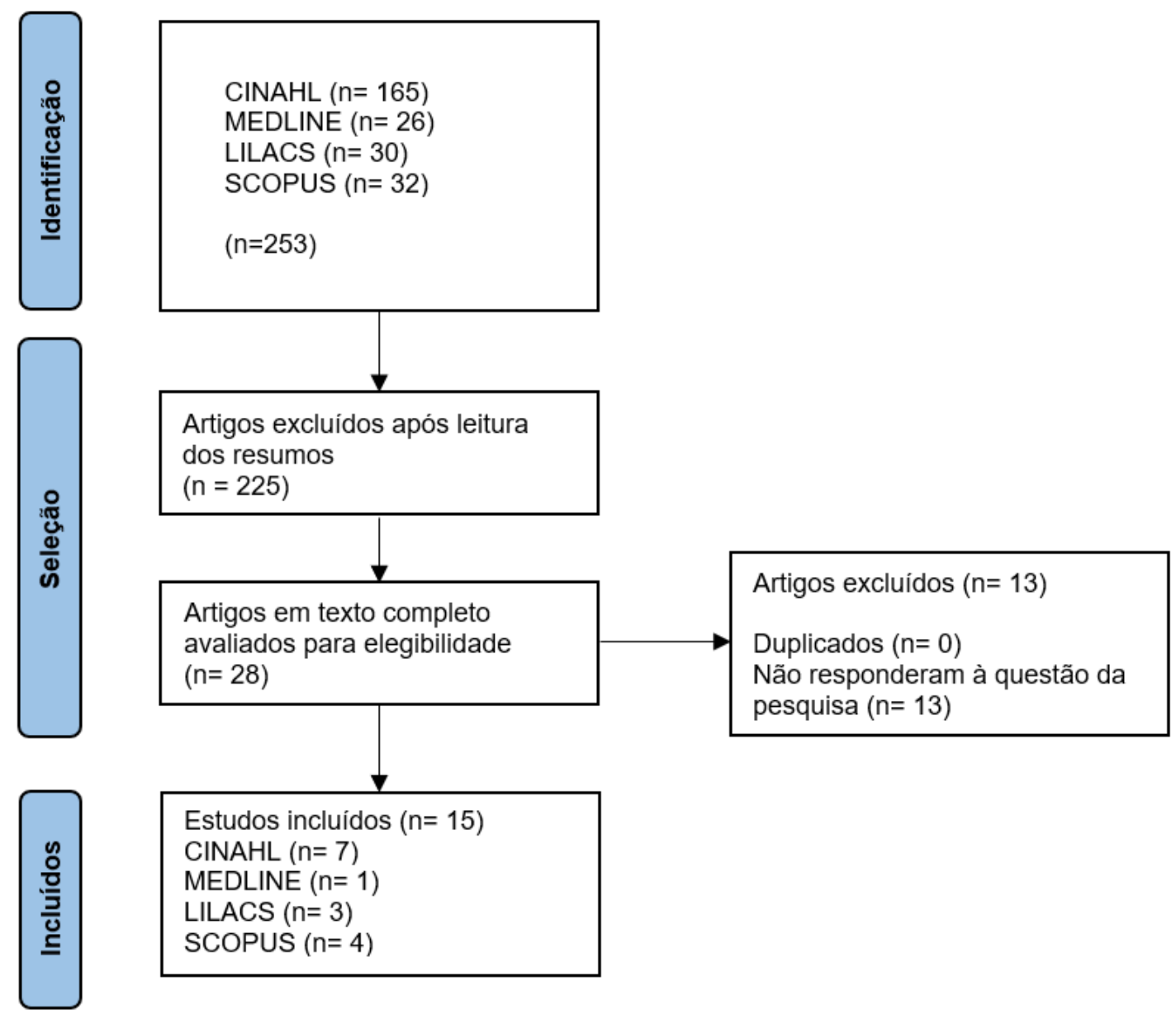

Fonte: Autores.

A análise dos dados foi realizada a partir de um quadro analítico que possibilitou reunir e sintetizar as informaçõeschave dos estudos. Foi utilizado o instrumento adaptado pelas pesquisadoras, contendo dados referentes à identificação do artigo (título, periódico, ano de publicação, local de busca, local do estudo); e identificação da pesquisa (objetivo, tipo de estudo, nível de evidência, resultados).

O trabalho de análise e integração da prática de enfermagem no campo da saúde mental constituiu em uma tarefa delicada e complexa, a qual foi sendo construída em um processo contínuo de reanálises do material. Associado a esse processo, aplicamos aos estudos o nível de evidência (NE) de acordo com a recomendação de Stetler et al. (1998).

O protocolo desta revisão sistemática está registrado na plataforma Open Science Framework (http://www.osf.io), sob o endereço https://doi.org/10.17605/OSF.IO/MP5NX

\section{Resultados}

Foram identificados 253 resultados no total. A figura 1 apresenta o fluxo e as etapas de seleção do material. Ao final deste processo 15 publicações foram incluídas para constituir a síntese deste estudo, que seguiram as etapas de identificação, triagem, elegibilidade e inclusão, atendendo aos critérios definidos anteriormente. O Quadro 1 apresenta a descrição dos estudos selecionados para esta revisão da literatura, especificando autoria, periódico, país, ano de publicação e nível de evidência. 
Quadro 1 - Publicações identificadas nas bases de dados de acordo com o ano, periódico, país de publicação - Niterói, RJ, Brasil, 2020.

\begin{tabular}{|c|c|c|c|c|c|c|}
\hline $\mathbf{N}^{0}$ & BASE & $\begin{array}{l}\text { AUTOR/ANO DE } \\
\text { PUBLICAÇÃO }\end{array}$ & PERIÓDICO & PAÍS & $\begin{array}{l}\text { TIPO } \\
\text { ESTUDO }\end{array}$ & $\begin{array}{l}\text { NÍVEL DE } \\
\text { EVIDÊNCI } \\
\text { A }\end{array}$ \\
\hline 1 & MEDLINE & $\begin{array}{l}\text { Bertram, } \\
\text { Narendorf, e } \\
\text { McMillen (2013) }\end{array}$ & $\begin{array}{l}\text { Archives } \\
\text { Psychiatric } \\
\text { Nursing }\end{array}$ & Estados Unidos & Descritivo & 4 \\
\hline 2 & LILACS & $\begin{array}{l}\text { Vargas, } \\
\text { Bittencourt, Rocha, } \\
\text { e Silva (2014) }\end{array}$ & $\begin{array}{l}\text { Revista Escola } \\
\text { Anna Nery }\end{array}$ & Brasil & Exploratório & 4 \\
\hline 3 & LILACS & Lima et al. (2014) & $\begin{array}{ll}\text { Revista } & \text { Escola } \\
\text { Anna Nery } & \end{array}$ & Brasil & Descritivo & 4 \\
\hline 4 & SCOPUS & $\begin{array}{l}\text { Farmakas, } \\
\text { Papastavrou, } \\
\text { Siskou, } \\
\text { Karayiannis, e } \\
\text { Theodorou (2014) }\end{array}$ & $\begin{array}{lr}\text { Journal } & \text { of } \\
\text { Psychiatric } & \text { and } \\
\text { Mental } & \text { Health } \\
\text { Nursing } & \end{array}$ & Estados Unidos & Descritivo & 4 \\
\hline 5 & SCOPUS & Allen (2016) & $\begin{array}{l}\text { Journal of the } \\
\text { American } \\
\text { Psychiatric Nurses } \\
\text { Association }\end{array}$ & Estados Unidos & Exploratório & 4 \\
\hline 6 & SCOPUS & $\begin{array}{l}\text { Pessoa Júnior, } \\
\text { Santos, } \\
\text { Clementino, } \\
\text { Nascimento, e } \\
\text { Miranda (2016) } \\
\end{array}$ & $\begin{array}{l}\text { Revista Texto \& } \\
\text { Contexto } \\
\text { Enfermagem }\end{array}$ & Brasil & Exploratório & 4 \\
\hline 7 & LILACS & $\begin{array}{l}\text { Vargas, Soares, } \\
\text { Ponce, e Oliveira } \\
(2017)\end{array}$ & $\begin{array}{l}\text { Revista Cogitare } \\
\text { Enfermagem }\end{array}$ & Brasil & Transversal & 3 \\
\hline 8 & CINAHL & $\begin{array}{l}\text { Damasceno e } \\
\text { Sousa (2018) }\end{array}$ & $\begin{array}{lr}\text { Revista } & \text { de } \\
\text { Enfermagem UFPE }\end{array}$ & Brasil & Descritivo & 4 \\
\hline 9 & CINAHL & $\begin{array}{l}\text { Lira, Silva, } \\
\text { Clementino, e } \\
\text { Barbosa (2018) }\end{array}$ & $\begin{array}{l}\text { Revista de } \\
\text { Enfermagem UFPE }\end{array}$ & Brasil & Descritivo & 4 \\
\hline 10 & CINAHL & $\begin{array}{l}\text { Mattos, Moll, } \\
\text { Souto, e Neves } \\
(2018)\end{array}$ & $\begin{array}{l}\text { Revista de } \\
\text { Enfermagem UFPE }\end{array}$ & Brasil & Descritivo & 4 \\
\hline 11 & CINAHL & $\begin{array}{l}\text { Santos, Pessoa } \\
\text { Junior, e Miranda } \\
\text { (2018) }\end{array}$ & $\begin{array}{l}\text { Revista Gaúcha de } \\
\text { Enfermagem }\end{array}$ & Brasil & Transversal & 3 \\
\hline 12 & CINAHL & $\begin{array}{l}\text { Moreira, Santos, } \\
\text { Azevedo, Araujo, e } \\
\text { Gusmao (2019) }\end{array}$ & $\begin{array}{l}\text { Enfermagem em } \\
\text { foco }\end{array}$ & Brasil & Transversal & 3 \\
\hline 13 & SCOPUS & $\begin{array}{l}\text { Salberg, } \\
\text { Bäckström, Röing, } \\
\text { e Öster (2019) }\end{array}$ & $\begin{array}{l}\text { Journal of Nursing } \\
\text { Management }\end{array}$ & Estados Unidos & $\begin{array}{l}\text { Fenomeno- } \\
\text { gráfico }\end{array}$ & 3 \\
\hline 14 & CINAHL & $\begin{array}{l}\text { Savarimuthu } \\
(2020)\end{array}$ & Journal of Nursing & Inglaterra & Reflexivo & 5 \\
\hline 15 & CINAHL & $\begin{array}{l}\text { Mesquita e } \\
\text { Tavares (2020) }\end{array}$ & $\begin{array}{l}\text { Enfermagem em } \\
\text { foco }\end{array}$ & Brasil & $\begin{array}{l}\text { Reflexão } \\
\text { teórica }\end{array}$ & 5 \\
\hline
\end{tabular}

Nota: UFPE - Universidade Federal de Pernambuco - Fonte: Autores. 
Entre os 15 estudos analisados, 10 foram publicados em português e cinco em inglês. Os artigos foram publicados em 11 diferentes periódicos. Revista de Enfermagem da Universidade Federal de Pernambuco (03); seguidas pela Escola Anna Nery Revista de Enfermagem (02) e Revista Enfermagem em Foco (02). Os demais periódicos foram a Revista Cogitare Enfermagem (01); Archives of Psychiatric Nursing (01); Journal Nursing Management (01); Journal of the American Psychiatric Nurses Association (01); Journal of Psychiatric and Mental Health Nursing (01); Revista Texto Contexto Enfermagem (01); Revista Gaúcha de Enfermagem (01); Darren. British Journal of Nursing (01).

No período de 10 anos, não houve nenhuma publicação de 2010 a 2012. O ano de 2018 obteve o maior número de trabalhos ( $n=4)$, seguidos dos anos de $2014(n=3), 2016(n=2), 2019(n=2)$ e $2020(n=2)$, enquanto nos anos 2013 e 2017 a frequência foi de um único artigo a cada ano. Apenas Vargas et al. (2014) e Vargas et al. (2017) publicou mais de um trabalho sobre o tema durante esse período. Somente dois artigos foram publicados por um único autor (Allen, 2016; Savarimuthu, 2020), todos os demais trabalhos tiveram sua autoria compartilhada com um ou mais autores.

Sobre 2018 ter sido o ano em que tivemos maior número de publicações, pode-se pensar no curso de especialização em grande escala oferecido por meio da parceria do Ministério da Saúde, através da Secretaria de Gestão do Trabalho e de Educação na Saúde (SGTES), da CGMAD e a da Universidade de Santa Catarina (UFSC). Foram ofertadas 300 vagas para formar enfermeiros especialistas em Saúde Mental. O curso foi concluído em julho de 2015 com 237 profissionais titulados, o que pode ter influenciado o interesse por essa temática e produzido publicações.

Com relação ao tipo de estudo, seis (40\%) são artigos descritivos. Os demais são estudos do tipo transversal (20\%), exploratório (20\%), reflexão (13\%) e fenomenográfico (7\%). Destes estudos, 10 (67\%) estão direcionados a descrever ou analisar as práticas e o cuidado de enfermagem na saúde mental; dois (13\%) se ocupam de traçar o perfil e descrever características dos profissionais e três (20\%) abordam um aspecto do processo de enfermagem, na perspectiva da saúde mental; seja a teoria, os diagnósticos ou a própria sistematização da assistência.

Dos artigos selecionados, nove (60\%) estão publicados em periódicos nacionais, todos em revistas de enfermagem, e seis (40\%) estão publicados em periódicos internacionais variados, nenhum com mais de uma publicação. Quanto ao recorte temporal de publicação, observou-se que os estudos são recentes com o intervalo entre 2013 e 2020, pois datam dos últimos 10 anos. Com nove artigos na língua portuguesa e, os demais, na língua inglesa.

No sentido de ampliar os resultados obtidos com os artigos, temos o Quadro 2, que nos oferece a análise do objetivo de cada publicação e o que podem ser identificados como resultados das práticas de enfermagem dos artigos selecionados.

Quadro 2 - Publicações identificadas, de acordo com o objetivo de publicação, e os principais resultados dos artigos relacionadas as práticas de enfermagem - Niterói, RJ, Brasil, 2020.

\begin{tabular}{|l|l|l|l|}
\hline $\begin{array}{l}\text { NOME DO } \\
\text { PERIÓDICO }\end{array}$ & $\begin{array}{l}\text { TÍTULO DA } \\
\text { PUBLICAÇãO }\end{array}$ & $\begin{array}{l}\text { ANÁLISE DO OBJETIVO DA } \\
\text { PUBLICAÇÃO }\end{array}$ & $\begin{array}{l}\text { IDENTIFICAÇÃO DOS RESULTADOS } \\
\text { DAS PRÁTICAS ENCONTRADAS NA } \\
\text { PUBLICAÇÃO }\end{array}$ \\
\hline $\begin{array}{l}\text { Archives } \\
\begin{array}{l}\text { Psychiatric } \\
\text { Nursing }\end{array}\end{array}$ & $\begin{array}{l}\text { Pioneering the } \\
\text { psychiatric nurse role } \\
\text { in foster care } \\
\text { (Bertram et al., 2013) }\end{array}$ & $\begin{array}{l}\text { Descreve os detalhes do trabalho do } \\
\text { enfermeiro psiquiátrico em uma } \\
\text { equipe multidisciplinar e aborda as } \\
\text { lacunas no cuidado que foram } \\
\text { identificadas em torno do tratamento } \\
\text { de saúde mental. }\end{array}$ & $\begin{array}{l}\text { - Executa um papel amplo no acolhimento } \\
\text { familiar para jovens mais velhos, identificando } \\
\text { sintomas que ultrapassavam os limites } \\
\text { diagnósticos e as apresentações clínicas que não } \\
\text { eram adequadamente contabilizadas pelos } \\
\text { critérios do DSM-IV; } \\
\text { - Aatuação capaz de fornecer um olhar aguçado } \\
\text { para processos e padrões relacionados ao } \\
\text { diagnóstico e prescrição psiquiátrica forneceu } \\
\text { uma perspectiva integrada sobre saúde física e } \\
\text { mental para uma população com comorbidades } \\
\text { complexas. }\end{array}$ \\
\hline
\end{tabular}




\begin{tabular}{|c|c|c|c|}
\hline $\begin{array}{l}\text { Revista Escola } \\
\text { Anna Nery }\end{array}$ & $\begin{array}{l}\text { Alcohol/drugs } \\
\text { community mental } \\
\text { health services: } \\
\text { insertion and } \\
\text { practices of nursing } \\
\text { professionals (Vargas } \\
\text { et al., 2014) (Vargas, } \\
\text { Bittencourt, Rocha, } \\
\text { \& Silva, 2014) }\end{array}$ & $\begin{array}{l}\text { Verifica a inserção e as práticas dos } \\
\text { profissionais de nível médio da equipe } \\
\text { de enfermagem nos CAPS álcool e } \\
\text { drogas. }\end{array}$ & $\begin{array}{l}\text { - Executam atividades referentes ao seu núcleo } \\
\text { específico de conhecimento, como: } \\
\text { administração e orientação medicamentosa, } \\
\text { realização de curativos e aferição de sinais } \\
\text { vitais; participam de oficinas e grupos } \\
\text { educativos; auxiliam na administração, } \\
\text { farmácia, na recepção, na cozinha, nas oficinas } \\
\text { como apoio; } \\
\text { - } 52,63 \% \text { dos entrevistados referiram não ter } \\
\text { recebido preparo no curso de formação para } \\
\text { executar o cuidado em saúde mental. }\end{array}$ \\
\hline $\begin{array}{l}\text { Revista Escola } \\
\text { Anna Nery }\end{array}$ & $\begin{array}{l}\text { Theoretical } \\
\text { references that guide } \\
\text { nursing practice in } \\
\text { mental health (Lima } \\
\text { et al., 2014) }\end{array}$ & $\begin{array}{l}\text { Identifica os referenciais teóricos que } \\
\text { norteiam a prática clínica de } \\
\text { enfermagem em saúde mental. }\end{array}$ & $\begin{array}{l}\text { - Referencial das teorias de enfermagem; } \\
\text { referencial biomédico; referencial das práticas } \\
\text { alternativas; referencial da política de redução } \\
\text { de danos; e o referencial da psicanálise. } \\
\text { - Há dificuldade por parte dos enfermeiros em } \\
\text { definir, quais os referenciais teóricos que } \\
\text { subsidiam sua atuação; } \\
\text { - Existe uma predominância do referencial } \\
\text { biomédico nos discursos dos enfermeiros. }\end{array}$ \\
\hline $\begin{array}{l}\text { Journal of } \\
\text { Psychiatric and } \\
\text { Mental Health } \\
\text { Nursing }\end{array}$ & $\begin{array}{l}\text { Challenges in mental } \\
\text { health nursing: } \\
\text { working in } \\
\text { institutional or } \\
\text { community settings? } \\
\text { (Farmakas et al., } \\
\text { 2014) }\end{array}$ & $\begin{array}{l}\text { Explora as percepções dos } \\
\text { enfermeiros de saúde mental em seu } \\
\text { ambiente de prática profissional e } \\
\text { examina as diferenças nas percepções } \\
\text { entre os enfermeiros que trabalham em } \\
\text { instituições e aqueles que atuam em } \\
\text { cuidados comunitários. }\end{array}$ & $\begin{array}{l}\text { - Enfermeiros que trabalham em hospital } \\
\text { psiquiátrico perceberam o ambiente de prática } \\
\text { profissional de forma mais negativo, e também } \\
\text { tiveram percepções de motivação, liderança e } \\
\text { autonomia na prática profissional menores do } \\
\text { que os que trabalhavam em atendimento } \\
\text { comunitário. }\end{array}$ \\
\hline $\begin{array}{l}\text { Journal of the } \\
\text { American } \\
\text { Psychiatric } \\
\text { Nurses } \\
\text { Association }\end{array}$ & $\begin{array}{l}\text { APNA Member } \\
\text { survey results: role of } \\
\text { psychiatric nurses in } \\
\text { hospital emergency } \\
\text { departments (Allen, } \\
\text { 2016) }\end{array}$ & $\begin{array}{l}\text { Descreve o papel e função da } \\
\text { enfermeira psiquiátrica nos } \\
\text { departamentos de emergência do } \\
\text { hospital e determina as necessidades } \\
\text { de treinamento e } \\
\text { competências. }\end{array}$ & $\begin{array}{l}\text { - Gestão de crise; } \\
\text { - Gestão de cuidados; } \\
\text { - Especialista/elo para os profissionais da } \\
\text { emergência. }\end{array}$ \\
\hline $\begin{array}{l}\text { Revista Texto } \\
\& \quad \text { Contexto } \\
\text { Enfermagem }\end{array}$ & $\begin{array}{l}\text { Mental } \\
\text { education }\end{array} \begin{array}{r}\text { and } \\
\text { professional } \\
\text { in the practice } \\
\text { hospital } \\
\text { Júnior et al., 2016) }\end{array}$ & $\begin{array}{l}\text { Identifica a opinião de profissionais } \\
\text { sobre formação em saúde mental e a } \\
\text { atuação profissional no âmbito do } \\
\text { hospital psiquiátrico. }\end{array}$ & $\begin{array}{l}\text { - Os profissionais apontam que a prática } \\
\text { profissional, no contexto do hospital } \\
\text { psiquiátrico, prioriza o atendimento individual } \\
\text { ao usuário e família, na perspectiva do } \\
\text { acolhimento e apoio emocional; } \\
\text { - Há preocupação dos profissionais com a } \\
\text { qualidade de vida e a inserção social dos } \\
\text { usuários atendidos; } \\
\text { - A maioria dos profissionais avaliou necessária } \\
\text { a Educação Permanente em Saúde Mental para } \\
\text { superação das dicotomias existentes entre o } \\
\text { saber e o fazer profissional. }\end{array}$ \\
\hline $\begin{array}{l}\text { Revista } \\
\text { Cogitare } \\
\text { Enfermagem }\end{array}$ & $\begin{array}{l}\text { Psychiatric urgency } \\
\text { and emergency care } \\
\text { nurses: an analysis of } \\
\text { their professional and } \\
\text { educational profile } \\
\text { (Vargas et al., 2017) }\end{array}$ & $\begin{array}{l}\text { Define o perfil profissional e } \\
\text { educacional de enfermeiros de } \\
\text { urgência e emergência psiquiátrica, } \\
\text { identificando sua associação com a } \\
\text { prática clínica. }\end{array}$ & $\begin{array}{l}\text { - A prática clínica apresentada foi maior nos } \\
\text { enfermeiros que possuíam especialização em } \\
\text { Psiquiatria e nos que atuavam em serviços de } \\
\text { porta aberta. } \\
\text { - Os enfermeiros que mais identificaram sua } \\
\text { prática clínica foram os formados por } \\
\text { universidades públicas e os que possuíam maior } \\
\text { tempo de experiência no campo. } \\
\text { - Profissionais do sexo masculino possuem } 9 \\
\text { vezes mais chances de executarem a prática } \\
\text { clínica em serviços de urgência e emergência em } \\
\text { psiquiatria. }\end{array}$ \\
\hline
\end{tabular}




\begin{tabular}{|c|c|c|c|}
\hline $\begin{array}{l}\text { Revista de } \\
\text { Enfermagem } \\
\text { UFPE }\end{array}$ & $\begin{array}{l}\text { Mental health care } \\
\text { for the elderly: the } \\
\text { nurse`s perception } \\
\text { (Damasceno \& } \\
\text { Sousa, 2018) }\end{array}$ & $\begin{array}{l}\text { Identifica as percepções dos } \\
\text { enfermeiros atuantes na atenção } \\
\text { primária à saúde sobre o cuidado de } \\
\text { saúde mental à pessoa idosa. }\end{array}$ & $\begin{array}{l}\text { - As práticas de cuidado às pessoas idosas com } \\
\text { adoecimento psíquico se organizam por meio de } \\
\text { consultas, visitas domiciliares e atividades } \\
\text { educativas que, por vezes, são permeadas pela } \\
\text { escuta das necessidades de saúde dos usuários e, } \\
\text { por outro lado, pela centralidade em exames e } \\
\text { procedimentos. }\end{array}$ \\
\hline $\begin{array}{l}\text { Revista de } \\
\text { Enfermagem } \\
\text { UFPE }\end{array}$ & $\begin{array}{l}\text { Between policies and } \\
\text { practices: therapeutic } \\
\text { activities based on } \\
\text { harm reduction (Lira } \\
\text { et al., 2018) }\end{array}$ & $\begin{array}{l}\text { Investiga as práticas terapêuticas } \\
\text { realizadas em Centros de Atenção } \\
\text { Psicossocial de Álcool e Drogas } \\
\text { (CAPSad) pautadas na política de } \\
\text { redução de danos. }\end{array}$ & $\begin{array}{l}\text { - A premissa da RD como fundamental para o } \\
\text { funcionamento do CAPS, baseada na não } \\
\text { preconização imediata da abstinência. O } \\
\text { trabalho é no sentido de: resgate da autoestima, } \\
\text { incentivo ao autocuidado, construção de projeto } \\
\text { de vida e os entornos coletivos como troca de } \\
\text { experiências (inclui a família); } \\
\text { - A equipe profissional é designada como o } \\
\text { principal elemento da operacionalização da RD. }\end{array}$ \\
\hline $\begin{array}{l}\text { Revista de } \\
\text { enfermagem } \\
\text { UFPE }\end{array}$ & $\begin{array}{lr}\text { Psychiatric } & \text { care } \\
\text { provided by } & \text { nursing } \\
\text { technicians } & \text { (Mattos } \\
\text { et al., 2018) } & \end{array}$ & $\begin{array}{l}\text { Investiga as práticas assistenciais dos } \\
\text { técnicos de Enfermagem às pessoas } \\
\text { com transtornos mentais. }\end{array}$ & $\begin{array}{l}\text { - Destacou-se a medicalização do cuidado e as } \\
\text { intervenções não medicamentosas centraram-se } \\
\text { na higienização, alimentação e sono; } \\
\text { - A conduta mais empática ocorria na alta } \\
\text { hospitalar quando se dialogava e organizava os } \\
\text { pertences do usuário e do acompanhante para } \\
\text { conduzi-los à portaria. }\end{array}$ \\
\hline $\begin{array}{l}\text { Revista Gaúcha } \\
\text { de Enfermagem }\end{array}$ & $\begin{array}{l}\text { Psychosocial care } \\
\text { network: adequacy of } \\
\text { roles and functions } \\
\text { performed by } \\
\text { professionals (Santos } \\
\text { et al., 2018) }\end{array}$ & $\begin{array}{l}\text { Verifica a adequação dos papéis e } \\
\text { funções desempenhados pelos } \\
\text { profissionais de nível superior nos } \\
\text { serviços da rede de atenção } \\
\text { psicossocial de uma capital do } \\
\text { Nordeste brasileiro. }\end{array}$ & $\begin{array}{l}\text { - Há uma adequação quanto aos atendimentos } \\
\text { individuais e a grupos de usuários, com } \\
\text { observação e registro do comportamento do } \\
\text { usuário e condutas terapêuticas no prontuário; } \\
\text { - No atendimento familiar, em relação a } \\
\text { abordagem utilizada no atendimento, o estudo } \\
\text { demonstrou que alguns profissionais esboçaram } \\
\text { respostas confusas, superficiais, mostrando, às } \\
\text { vezes, desconhecimento e sugerindo a } \\
\text { realização de um trabalho de maneira empírica, } \\
\text { sem qualquer embasamento cientifico; } \\
\text { - Encontrou-se um nível de inadequação } \\
\text { referente a realização de grupos de familiares } \\
\text { entre os serviços e profissionais pesquisados. }\end{array}$ \\
\hline $\begin{array}{l}\text { Enfermagem } \\
\text { em foco }\end{array}$ & $\begin{array}{l}\text { Nursing diagnoses } \\
\text { identified in alcohol } \\
\text { and other drug users } \\
\text { (Moreira et al., 2019) }\end{array}$ & $\begin{array}{l}\text { Identifica os principais diagnósticos } \\
\text { de enfermagem em usuários de álcool } \\
\text { e outras drogas, acompanhados em um } \\
\text { Centro de Atenção Psicossocial. }\end{array}$ & $\begin{array}{l}\text { - Os diagnósticos de enfermagem foram } \\
\text { distribuídos conforme as Necessidades } \\
\text { psicoespirituais, psicossociais ou } \\
\text { psicobiológicas, baseadas na Teoria das } \\
\text { Necessidades Humanas Básicas de Wanda } \\
\text { Aguiar Horta; } \\
\text { - Obtiveram-se } 60 \text { diferentes DEs, com } \\
\text { predomínio daqueles relacionados às } \\
\text { Necessidades Psicobiológicas; } \\
\text { - Referente às Necessidades Psicoespirituais, o } \\
\text { principal DE identificado foi "crença religiosa, } \\
\text { positiva"; } \\
\text { - No que refere às Necessidades Psicossociais, } \\
\text { todos os principais DEs são referentes à } \\
\text { Necessidade de Aprendizagem, sociabilidade, } \\
\text { recreação e lazer; } \\
\text { - No que se refere aos DEs "Conhecimento, } \\
\text { Prejudicado' e "Falta de conhecimento sobre } \\
\text { doença", ambos mantêm íntima relação com o } \\
\text { nível de escolaridade; } \\
\text { - Na Necessidade "Sexualidade e Reprodução" } \\
\text { os DEs frequentes foram "Processo sexual, }\end{array}$ \\
\hline
\end{tabular}




\begin{tabular}{|c|c|c|c|}
\hline & & & $\begin{array}{l}\text { preservado" e "Comportamento sexual, } \\
\text { problemático"; } \\
\text { - Em relação as Necessidades "Sono e repouso", } \\
\text { "Cuidado corporal" e "Terapêutica", } \\
\text { identificou-se com frequência os DEs "Insônia", } \\
\text { "Padrão de Higiene oral, inadequado" e "Adesão } \\
\text { ao regime medicamentoso". }\end{array}$ \\
\hline $\begin{array}{l}\text { Journal of } \\
\text { Nursing } \\
\text { Management }\end{array}$ & $\begin{array}{l}\text { Ways of } \\
\text { understanding } \\
\text { nursing in psychiatric } \\
\text { inpatient care - A } \\
\text { phenomenographic } \\
\text { study (Salberg et al., } \\
\text { 2019) }\end{array}$ & $\begin{array}{l}\text { Descreve as formas como a equipe de } \\
\text { enfermagem da internação psiquiátrica } \\
\text { compreende a enfermagem, no } \\
\text { atendimento psiquiátrico. }\end{array}$ & $\begin{array}{l}\text { - A enfermagem é responder a sintomas e } \\
\text { comportamentos; } \\
\text { - Enfermagem: é planejar e tratar; } \\
\text { - Enfermagem é apoiar seres humanos } \\
\text { vulneráveis, } \\
\text { - Enfermagem é motivar usuários e } \\
\text { - Enfermagem é ter uma perspectiva holística no } \\
\text { tratamento de doenças mentais. }\end{array}$ \\
\hline $\begin{array}{l}\text { Journal of } \\
\text { Nursing }\end{array}$ & $\begin{array}{l}\text { The potential role of } \\
\text { nurses in leading } \\
\text { positive behaviour } \\
\text { support } \\
\text { (Savarimuthu, 2020) }\end{array}$ & $\begin{array}{l}\text { Apresenta o papel da enfermagem } \\
\text { dentro da metodologia de suporte de } \\
\text { comportamento positivo PBS } \\
\text { (positive behaviour support), } \\
\text { considerada uma intervenção preferida } \\
\text { na gestão de comportamentos } \\
\text { desafiadores na deficiência de } \\
\text { aprendizagem e nos serviços de saúde } \\
\text { mental. }\end{array}$ & $\begin{array}{l}\text { - O perfil profissional da enfermagem se } \\
\text { caracteriza pela liderança. O artigo sugere uma } \\
\text { postura mais expressiva da enfermagem dentro } \\
\text { da PBS, no que diz respeito a liderança; } \\
\text { - Apresenta os enfermeiros como elos na } \\
\text { comunicação entre a equipe multiprofissional } \\
\text { em saúde mental, o que facilitaria a } \\
\text { implementação da metodologia assistencial. }\end{array}$ \\
\hline $\begin{array}{l}\text { Enfermagem } \\
\text { em foco }\end{array}$ & $\begin{array}{l}\text { Systematization of } \\
\text { nursing and clinical } \\
\text { assistance expanded: } \\
\text { challenges for mental } \\
\text { health education } \\
\text { (Mesquita \& Tavares, } \\
2020 \text { ) }\end{array}$ & $\begin{array}{l}\text { Reflete sobre os desafios engendrados } \\
\text { pela proposta da SAE e da Clínica } \\
\text { Ampliada (CA) para o ensino de } \\
\text { enfermagem em saúde mental. }\end{array}$ & $\begin{array}{l}\text { - Nos serviços de saúde mental, a SAE não é um } \\
\text { método usual de trabalho adotado pelos } \\
\text { enfermeiros; } \\
\text { - Escassez de literatura sobre a aplicação, } \\
\text { avaliação e eficácia do PE em saúde mental. As } \\
\text { etapas de histórico e planejamento dos cuidados } \\
\text { de enfermagem são as mais executadas, e tidas } \\
\text { como contribuição do enfermeiro para a } \\
\text { construção do PTS e sua inserção na equipe } \\
\text { multiprofissional; } \\
\text { - A experiência por parte de enfermeiros na } \\
\text { utilização da perspectiva de Peplau (cuidado é } \\
\text { singular e o seu foco é a relação terapêutica) } \\
\text { sobre o uso da relação terapêtica como centro } \\
\text { das intervenções; } \\
\text { - A clínica ampliada como referencial de } \\
\text { construção de cuidado integral com centralidade } \\
\text { no sujeito e a participação da enfermagem ainda } \\
\text { não consolidado; } \\
\text { - Enfermagem, 50\% da força de trabalho, porém } \\
\text { considera sua atuação como "pseudo-clínica". }\end{array}$ \\
\hline
\end{tabular}

Fonte: Autores.

A partir das informações acimas extraídas dos artigos, foi elaborado um resumo das práticas de enfermagem que puderam ser identificadas e o número de vezes que foram citadas nos trabalhos, organizadas no Quadro 3. 
Quadro 3 - Demonstração da síntese das funções desempenhadas pela enfermagem.

\begin{tabular}{|c|c|c|}
\hline PRÁTICA DE ENFERMAGEM & $\begin{array}{l}N^{\circ} \text { DE VEZES } \\
\text { QUE } \\
\text { APARECEU } \\
\text { NOS ESTUDOS }\end{array}$ & PERIÓDICO / TÍTULO DOS ESTUDOS \\
\hline $\begin{array}{l}\text { Medicação: responder ao cuidado a partir da } \\
\text { medicalização; orientar, administrar e } \\
\text { observar quanto a adesão medicamentosa. }\end{array}$ & 3 & $\begin{array}{l}\text { Revista Escola Anna Nery / Alcohol/drugs community mental } \\
\text { health services: insertion and practices of nursing professionals. }\end{array}$ \\
\hline Realizar curativos e aferição de sinais vitais. & 2 & $\begin{array}{l}\text { Revista Escola Anna Nery / Alcohol/drugs community mental } \\
\text { health services: insertion and practices of nursing professionals. } \\
\text { Revista de Enfermagem UFPE/ Mental health care for the } \\
\text { elderly: the nurse`s perception. }\end{array}$ \\
\hline Responder a sintomas e comportamentos. & 1 & $\begin{array}{l}\text { Journal of Nursing Management / Ways of understanding } \\
\text { nursing in psychiatric inpatient care - } \\
\text { A phenomenographic study. }\end{array}$ \\
\hline Higienização, alimentação e sono, & 2 & $\begin{array}{l}\text { Enfermagem em foco / Nursing diagnoses identified in alcohol } \\
\text { and other drug users. } \\
\text { Revista de enfermagem UFPE/ Psychiatric care provided by } \\
\text { nursing technicians. }\end{array}$ \\
\hline $\begin{array}{l}\text { Elos na comunicação entre a equipe } \\
\text { multiprofissional. }\end{array}$ & 1 & $\begin{array}{l}\text { Journal of Nursing/ The potential role of nurses in leading } \\
\text { positive behaviour support. }\end{array}$ \\
\hline Participar de oficinas. & 1 & $\begin{array}{l}\text { Journal of Nursing/ The potential role of nurses in leading } \\
\text { positive behaviour support. }\end{array}$ \\
\hline Participar de grupos educativos. & 2 & $\begin{array}{l}\text { Journal of Nursing/ The potential role of nurses in leading } \\
\text { positive behaviour support. } \\
\text { Revista de Enfermagem UFPE/ Mental health care for the } \\
\text { elderly: the nurse`s perception. }\end{array}$ \\
\hline $\begin{array}{l}\text { Auxiliar na administração, farmácia, na } \\
\text { recepção e na hora do almoço no CAPS. }\end{array}$ & 1 & $\begin{array}{l}\text { Revista Escola Anna Nery / Alcohol/drugs community mental } \\
\text { health services: insertion and practices of nursing professionals. }\end{array}$ \\
\hline Realizar acolhimento ao usuário/família. & 2 & $\begin{array}{l}\text { Archives of Psychiatric Nursing / Pioneering the Psychiatric } \\
\text { Nurse Role in Foster Care. } \\
\text { Revista Texto \& Contexto Enfermagem / Mental health } \\
\text { education and professional practice in the psychiatric hospital. }\end{array}$ \\
\hline Realizar consultas e visitas domiciliares. & 1 & $\begin{array}{l}\text { Revista de Enfermagem UFPE / Mental health care for the } \\
\text { elderly: the nurse`s perception. }\end{array}$ \\
\hline $\begin{array}{l}\text { Auxiliar no resgate da autoestima; incentivar } \\
\text { ao autocuidado e motivar o usuário. }\end{array}$ & 2 & $\begin{array}{l}\text { Journal of Nursing Management / Ways of understanding } \\
\text { nursing in psychiatric inpatient care - } \\
\text { A phenomenographic study. } \\
\text { Revista de Enfermagem UFPE / Between polices and practices: } \\
\text { therapeutic activities based on harm reduction. }\end{array}$ \\
\hline $\begin{array}{l}\text { Auxiliar na construção de projeto de vida e os } \\
\text { entornos coletivos como troca de experiências, } \\
\text { incluindo a família. }\end{array}$ & 2 & $\begin{array}{l}\text { Revista de Enfermagem UFPE / Between polices and practices: } \\
\text { therapeutic activities based on harm reduction. } \\
\text { Enfermagem em foco / Nursing diagnoses identified in alcohol } \\
\text { and other drug users. }\end{array}$ \\
\hline $\begin{array}{l}\text { Operacionalizar o trabalho na ótica da redução } \\
\text { de danos. }\end{array}$ & 1 & $\begin{array}{l}\text { Revista de Enfermagem UFPE / Between polices and practices: } \\
\text { therapeutic activities based on harm reduction. }\end{array}$ \\
\hline Planejar e tratar. & 1 & $\begin{array}{l}\text { Journal of Nursing Management/ Ways of understanding nursing } \\
\text { in psychiatric inpatient care - }\end{array}$ \\
\hline
\end{tabular}




\begin{tabular}{|l|l|l|}
\hline & & A phenomenographic study. \\
\hline $\begin{array}{l}\text { Realizar um cuidado centrado na relação } \\
\text { terapêutica. }\end{array}$ & 1 & $\begin{array}{l}\text { Enfermagem em foco / Systematization of nursing and clinical } \\
\text { assistance expanded: challenges for mental health education. }\end{array}$ \\
\hline
\end{tabular}

Fonte: Autores.

Dos 15 artigos analisados, foi possível extrair o total de 15 atividades práticas, o que demonstra de fato um leque amplo no papel compreendido dessa profissão.

A assistência junto a medicação dos usuários foi a função mais citadas pelos autores, aparecendo em três estudos.

\section{Discussão}

Essa revisão sistemática da literatura revelou que as práticas de enfermagem desenvolvidas para o cuidado com a pessoa em sofrimento psíquico foram, principalmente, a administração de medicamentos, aferição de sinais vitais, higiene, alimentação, participação em oficinas, visita domiciliar, atividades com a família, acolhimento, atividades na ótica da redução de danos, trabalho em equipe com foco na singularidade do usuário, com projetos terapêuticos, atividades de promoção da autoestima e autocuidado, participação em grupos educativos e ações de planejamento do cuidado, mas ainda identificam-se outros tipos de práticas. Com isso, percebe-se que as práticas e o cuidado de enfermagem em saúde mental mantêm atividades próprias das teorias de necessidades humanas básicas, as quais estão sendo acrescidas de ações que no seu desenvolvimento incorporam tecnologias originadas da educação e da ciência do cuidado em enfermagem. Contudo, são necessários mais investimentos na pesquisa, com vistas a ampliar as possibilidades de intervenção para a prática clínica de enfermagem em saúde mental.

Entende-se que o momento atual da prática de enfermagem em saúde mental caracteriza-se pela transição entre uma prática hospitalar, com o foco na contenção e na vigilância do comportamento dos "doentes mentais", e à incorporação de princípios novos e desafiadores, em busca de uma assistência interdisciplinar, porosa às contingências dos sujeitos envolvidos em cada momento e contexto, superando assim a perspectiva disciplinar. É, por isso, um período crítico para a profissão e favorável para o conhecimento e análise do processo de trabalho nessa área (Alves \& Oliveira, 2010).

Identificou-se, entre os artigos incluídos nesta revisão que a administração de medicações dos usuários (Carvalho \& Felli, 2006; Paes, Maftum, \& Mantovani, 2010) está presente dentre as atividades de enfermagem, contudo os autores a delimitam como uma função mecânica, sendo também associada à higienização, alimentação e sono como principais atividades desenvolvidas no ambiente hospitalar pelos técnicos de enfermagem (Mattos, Moll, Souto, \& Neves, 2018). Atividade esta associada a interação com o familiar e usuário durante a alta hospitalar.

Em contrapartida, em outro estudo (Vargas, Bittencourt, Rocha, \& Silva, 2014) verificou-se que os profissionais de nível médio da equipe de enfermagem em Centro de Atenção Psicossocial executavam além de atividades referentes ao seu núcleo específico de conhecimento, como: administração, orientação medicamentosa, realização de curativos e aferição de sinais vitais; também participavam em oficinas e grupos educativos; na recepção, na cozinha e no grupo de caminhada.

Aqui destacamos a complexidade da prática da enfermagem em Saúde Mental, quer seja no hospital ou em outros dispositivos que passam a incorporar elementos da constituição subjetiva do sujeito em sua prática. Nessa mesma perspectiva Loyola (2017) destaca o cuidado de enfermagem em saúde mental, como um saber que sai do saber exposto - de teorias e técnicas, para o saber suposto - o sujeito como potência.

O acolhimento familiar é reconhecido como papel da enfermeira psiquiátrica (Bertram, Narendorf, \& McMillen, 2013; Pessoa Júnior, Santos, Clementino, Nascimento, \& Miranda, 2016; Salberg, Bäckström, Röing, \& Öster, 2019), como a possibilidade de identificar sintomas que ultrapassavam os limites diagnósticos e as apresentações clínicas não contabilizadas pelos critérios do DSM-IV; além de fornecer uma perspectiva integrada sobre saúde física e mental para uma população com 
comorbidades complexas. Nesse processo há a preocupação dos profissionais tanto com a qualidade de vida quanto com a inserção social e emocional dos usuários atendidos. Essa perspectiva holística no tratamento como inerente à prática de enfermagem em saúde mental está presente na internação psiquiátrica da equipe de enfermagem, como função no atendimento.

Entretanto, na prática clínica, o acolhimento enfrenta problemas em sua efetivação na área de saúde mental. Ocorre uma inadequação na forma de realização de atendimentos aos familiares, nas dinâmicas de grupos de família, em relação a abordagem utilizada durante o atendimento (Santos, Pessoa Junior, \& Miranda, 2018). O que pode ser exemplificado com a conduta de alguns profissionais que esboçaram respostas confusas, superficiais, além do medo de heteroagressividade, mostrando às vezes, desconhecimento e sugerindo a realização de um trabalho de maneira empírica, sem qualquer embasamento científico e atualização em saúde mental.

Apesar das questões identificadas com o acolhimento, este dispositivo mantém-se como viabilizador do encontro entre usuário e profissional no relacionamento interpessoal e formação de vínculo, proporcionando a escuta e a empatia, possibilitando, assim, a qualificação da assistência dos profissionais de enfermagem. Podemos incluir o acolhimento como um espaço coletivo que atravessa o cotidiano dos trabalhadores e o processo de cuidado dos usuários e seus familiares, promovendo também relações de trabalho mais horizontais, flexíveis e decentralizadas, o que pode trazer a sensação de maior autonomia, liderança e satisfação por parte desses profissionais de enfermagem.

Entre as práticas já identificadas destacamos também a gestão de crise e a gestão de cuidados (Bertram et al., 2013; Salberg et al., 2019) além da atividade de construir um elo com as demais profissões do campo da saúde mental em hospitais. Essas ações estão relacionadas à ação dos enfermeiros e são práticas de planejamento das atividades, que incluem a melhoraria da prática assistencial, possibilitando melhores intervenções e, consequentemente, melhores resultados: atuar nos sintomas, responder aos comportamentos dos usuários, apoiando e motivando-os quando estão em situações de maior vulnerabilidade.

A prática, quando associada ao perfil profissional e educacional (Farmakas, Papastavrou, Siskou, Karayiannis, \& Theodorou, 2014; Vargas, Soares, Ponce, \& Oliveira, 2017), revela alguns pontos importantes como: profissionais do sexo masculino atuando em emergências e urgências psiquiátricas em maior número; enfermeiros formados por universidades públicas, com maior tempo de experiência e com especialização em Psiquiatria. Um perfil que está presente no ambiente hospitalar e nos serviços de porta aberta. Em relação aos aspectos educacionais (Farmakas et al., 2014; Vargas, Bittencourt, Rocha, \& Silva, 2014; D. Vargas et al., 2017), os estudos afirmam que a mercantilização do sistema, com base nas concepções neoliberais, impõe um modelo de sociedade na qual a educação passa a ser reduzida a um bem de consumo, afetando a qualidade da formação oferecida, e isso fica mais evidente nas instituições privadas de ensino.

O conteúdo do cuidado de enfermagem (Fritsch, Jacobus, \& Vitelli, 2020; Rodrigues, Santos, \& Spriccigo, 2012) presente nas competências e habilidades da disciplina/módulo de enfermagem psiquiátrica e saúde mental, de modo geral, nos cursos, são incipientes. Uma realidade facilitada por parte, pela não definição na legislação brasileira da exposição de conteúdos essenciais e/ou obrigatórios sobre a enfermagem em saúde mental/psiquiátrica, dentro dos currículos de graduação em Enfermagem generalista (Lei $\left.{ }^{\circ} 7.498,1986\right)$.

A análise dos artigos permite apontar uma convergência teórica, de referenciais que se baseiam nas relações interpessoais para o cuidado de enfermagem na saúde mental, como as teorias Peplau (1962) e Travelbee (1969) em torno da compreensão de que o enfermeiro em serviços de saúde mental tem esse papel de "agente terapêutico", cujo objetivo fundamental é auxiliar o usuário a aceitar a si próprio e a melhorar as suas relações pessoais, motivá-lo e ajuda-lo na busca de novos sentidos e restauração da sua autonomia (Carrara, 2015). Dessa maneira, o relacionamento terapêutico é um instrumento de cuidado importante para a enfermagem na saúde mental que permite a reinserção e organização dos sujeitos que sofrem psiquicamente. Por meio do Processo de Enfermagem (PE), objetiva-se formular o cuidado apostando na relação terapêutica construída com o enfermeiro. Um processo com os principais diagnósticos e a sistematização da assistência de Enfermagem na área de saúde 
mental (Lima et al., 2014; Mesquita \& Tavares, 2020).

Também tiveram aqueles que se ocuparam em abordar as principais teorias utilizadas no campo, identificar os diagnósticos mais usados pelos enfermeiros e refletir sobre a realidade da Sistematização da Assistência em Enfermagem (SAE) na saúde mental (Lima et al., 2014; Mesquita \& Tavares, 2020; Moreira, Santos, Azevedo, Araujo, \& Gusmao, 2019).

Os estudos incluídos no trabalho de Lima et al. (2014) constataram que há dificuldade por parte dos enfermeiros em definir quais os referenciais teóricos que subsidiam sua atuação. Indo das teorias de enfermagem; referencial das práticas alternativas; referencial da política de redução de danos; referencial da psicanálise e o referencial biomédico; sendo esse último, predominante nos discursos dos enfermeiros. Sobre isso, Carrara (2015) afirma que há necessidade também de haver perante os profissionais de saúde, principalmente a enfermagem a base em conhecimentos teóricos que possam ser utilizada na prática dos relacionamentos terapêuticos do enfermeiro, permitindo a proximidades com os pacientes para que os mesmos não tenham só a terapêutica dos psicofármacos. Ou seja, a escolha do referencial teórico norteia a perspectiva do cuidado que se pretende empregar, de modo que influencia diretamente no foco da sua assistência em relação às necessidades do usuário. Nesse sentido a etapa da elaboração dos diagnósticos precisam estar em consonância com a teoria adotada.

$\mathrm{O}$ uso das teorias de enfermagem como referenciais teóricos importantes do núcleo de conhecimento da profissão são fundamentais para a garantia da reorientação do modelo de assistência nesse campo (Moreira et al., 2019; Oliveira et al., 2020). Atreladas a elas, teorias interdisciplinares, como a psicanálise, têm contribuído para o cuidado ao usuário com sofrimento psíquico (Oliveira et al., 2020). Assim, concluimos que sustenta-se uma prática de enfermagem embasada nas referidas referências científicas seja necessária para garantir a qualidade da assistência de enfermagem na saúde mental, proporcionando, como consequência, reconhecimento e visibilidade à profissão.

Os achados sobre as muitas funções executadas pelos enfermeiros nos convidam a refletir sobre a falta de especificidade no cuidado em saúde mental. Concordando, portanto, com Paes (2010) que diz que há “[...] falta de especificidade no cuidado ao usuário com morbidade clínico-psiquiátrica e que existe maior ênfase no conforto e nas técnicas do cotidiano de enfermagem, como administração de medicamentos, auxílio na higiene e deambulação" (p. 283) Sobre a associação do tempo de experiência com uma maior prática clínica também evidenciada no momento do cuidado existe um conhecimento que vem da experiência, passa pelo corpo, não se resumindo a um saber racional, pois a experiência é "isso que me passa" (Larrosa, 2011, p. 21). Tem se então algo externo a mim, alheio e sempre singular, que não pode ser previamente capturado, mas que o lugar da experiência sou eu.

Percebe-se então, que as práticas de enfermagem são diversas e se diferem pelas peculiaridades dos espaços onde elas se dão, sejam em serviços hospitalares ou outros dispositivos; seja pela especificidade do profissional envolvido, podendo ser enfermeiro ou técnico de enfermagem.

A estrutura do ambiente hospitalar configura-se através do trabalho hierarquizado , ações isoladas e protocolos rígidos em um aparato propício para a normatização do cuidado, onde o núcleo do trabalho mais duro, menos flexível às reais necessidades do pacientes, tende a prevalecer em detrimento das práticas capazes de acolher as diversas dimensões da vida e as suas singularidades(Muniz, 2016). Já os Caps, a própria proposta do serviço( portas abertas, convivência, oficinas, assembleias, música, arte, atividades diversas, atendimentos fora dos espaços de consultório, entre outros), e a sua complexidade colocam como necessário à disposição dos profissionais para se deslocarem, estarem abertos a novas experimentação do cuidado, um cuidado que extrapola as normas e vaza das tradicionais salas de atendimentos e até mesmo dos muros desses serviços (Ferreira, Sampaio, Souza, Oliveira, \& Gomes, 2017).

É também no ambiente hospitalar, no que tange a área da psiquiatria e saúde mental, que se encontram muitos profissionais de enfermagem com anos de profissão, o que pode representar uma maior resistência às mudança, ao mesmo passo que também carregam consigo experiência acumuladas, que não devem ser desconsideradas, mesmo que se faça necessário a 
reconfiguração dos regimes de verdades que trazem com eles (Laker, Cella, Callard, \& Wykes, 2019).

Nesse contexto, que se torna imprescindível pensar em formas de intervenção em serviço, a partir de processos autoanalíticos, em que a velha prática é analisada, podendo ser descontruída, dando lugar a possibilidade de novas formas de cuidar.

A despeito disso, Franco e Merhy (2013) afirmam que os processos de Educação Permanente configuram-se como uma proposta que vai ao encontro dessas demandas: a formação de novos saberes associada a um processo de subjetivação.

Deste modo, Tavares, Silveira, Lima, Ferreira, e Santos (2015) reforçam a aposta na formação baseada numa abordagem de ensino mais libertadora, criativa e reflexiva, capaz de propiciar aos enfermeiros, conhecimentos, habilidade e atitudes condizentes com as expectativas da profissão e da sociedade.

Por fim, este estudo traz significativas contribuições para a prática de enfermagem na área da saúde mental, por meio do levantamento de práticas profissionais dessa categoria, demonstrando as ações de enfermagem nesse campo, fornecendo, assim, parâmetro para se avaliar se estão em direção aos preceitos da Reforma Psiquiátrica. Com isso, possibilita o (re)direcionamento e o planejamento das ações de Educação Permanente em Saúde. Ao trazer como proposta analisar, compreender e pensar os modos operantes de uma das maiores forças de trabalho, no que diz respeito a possibilidade de produzirem o seu cuidado, dentro dos dispositivos de saúde mental e de tratamento do SUS, contribui-se com a melhora da assistência prestada aos usuários com transtornos mentais, e representa uma forma de participação na Reforma Psiquiátrica.

Identificou-se a lacuna de produções relacionados à temática explorada, e por se tratar de uma revisão integrativa, os resultados retratam apenas a realidade investigada. Tem-se como limitação um número baseado em amostras pequena de estudos na área, que pode estar relacionado com a dificuldade em lidar com a clínica da saúde mental e o próprio desinteresse por esse tipo de atendimento (Sousa, Costa, \& Jorge, 2019).

\section{Conclusão}

O panorama encontrado indicou que a temática das práticas de enfermagem em saúde mental foi abordada de diferentes maneiras pelos artigos. Pôde-se perceber que os autores se preocuparam ora em traçar um perfil desses profissionais em relação às suas práticas clínicas, ora em descrever as atividades e ações desempenhadas pelo enfermeiro ou técnico/auxiliar, chegando a diferenciá-las e compará-las nos diversos serviços da rede de saúde mental.

A execução das práticas do enfermeiro em saúde mental, deve ser encarada como desafio que ultrapasse o padrão medicamentoso, e que crie propostas de cuidado com foco no usuário. Esse cuidado pautado no sujeito como potência mostrouse presente na dinâmica de trabalho dos CAPS, pela sua proposta de serviço substitutivo às práticas asilares da organização hospitalocêntrica, investindo para isso, na produção de cuidados que extrapolem o campo biomédico, em direção a uma proposta de mudança no imaginário social por meio de ações comunitárias e territoriais, proporcionando o direito à moradia, ao trabalho e ao lazer dos usuários. O território, portanto, como produtor de conexões que viabilizam a produção de vida dos sujeitos (Barbosa et al., 2020).

Proposta essa, que convoca para uma configuração diferenciada das relações de trabalho tradicionais encontradas nos hospitais (relações bem hierarquizadas, pautadas em poder, de domínio e submissão) entre os profissionais e desses com os usuários, em uma relação interpessoal, em que a prática da enfermagem esteja ancorada.

Configura-se, portanto, de grande relevância que a atuação do enfermeiro em saúde mental se sensibilize para as propostas exigidas na atual política de saúde mental, de modo que as dificuldades encontradas para a transformação dessa prática, sejam tomadas como alvo dos programas de formação em saúde, como a Educação Permanente. 


\section{Referências}

Allen, D. E. (2016). APNA member survey results: Role of psychiatric nurses in hospital emergency departments. Journal of the American Psychiatric Nurses Association, 22(3), 245-247. doi:10.1177/1078390316644325

Alves, M., \& Oliveira, R. M. P. (2010). Psychiatric nursing: Speech the ideal and practice the real. Escola Anna Nery, 14(1), 64-70. doi:10.1590/S141481452010000100010

Barbosa, A. S., Nascimento, C. V., Lucas B. S. Dias, L. B. S., Santo, T. B. E., Chaves, R. S. C., \& Fernandes, T. C. (2020). Processo de trabalho e cuidado em saúde mental no Centro de Atenção Psicossocial da UERJ na pandemia de COVID-19. Brazilian Journal of Health and Biomedical Sciences, 19(1). doi: $10.12957 /$ bjhbs. 2020.53527

Bertram, J. E., Narendorf, S. C., \& McMillen, J. C. (2013). Pioneering the psychiatric nurse role in foster care. Archives of Psychiatric Nursing, 27(6), 285-292. doi:10.1016/j.apnu.2013.09.003

Carrara, G. L. R. (2015). Humanized nursing care in mental health: A review of the literature. Revista Fafibe On-Line, 8(1), 86-107. Retrieved from https://www.unifafibe.com.br/revistasonline/arquivos/revistafafibeonline/sumario/36/30102015183642.pdf

Carvalho, M. B., \& Felli, V. E. A. (2006). Psychiatric nursing work and workers' health problems. Revista Latino-Americana de Enfermagem, 14(1), 61-69. doi:10.1590/s0104-11692006000100009

Cieto, B. B., Garbuio, D. C., Camargo, V. B., \& Napoleão, A. A. (2014). Nursing resources and innovations for hospital discharge: An integrative review. Revista Mineira de Enfermagem, 18(3), 752-757. doi:10.5935/1415-2762.20140055

Costa, J. M., Moraes Filho, I. M., \& Souza, S. A. N. (2019). The perception of the nursing team through psychiatric emergencies. Revista de Iniciação Científica e Extensão, 2(1), 15-23. Retrieved from https://revistasfacesa.senaaires.com.br/index.php/iniciacao-cientifica/article/view/137/91

Damasceno, V. C., \& Sousa, F. S. P. (2018). Mental health care for the elderly: The nurse's perception. Journal of Nursing UFPE, 12(10), $2710-2716$. doi:10.5205/1981-8963-v12i10a234647p2710-2716-2018

Farmakas, A., Papastavrou, E., Siskou, O., Karayiannis, G., \& Theodorou, M. (2014). Challenges in mental health nursing: Working in institutional or community settings? Journal of Psychiatric \& Mental Health Nursing, 21(1), 39-45. doi:10.1111/jpm.12045

Ferreira, T. P. S., Sampaio, J., Souza, A. C. N., Oliveira, D. L., \& Gomes, L. B. (2017). Produção do cuidado em Saúde Mental: desafios para além dos muros institucionais. Interface - Comunicação, Saúde, Educação, 21(61), 373-384. doi:10.1590/1807-57622016.0139

Franco, T. B., \& Merhy, E. E. (2013). Trabalho, produção do cuidado e subjetividade em saúde: Textos reunidos (1st ed.). Sāo Paulo: Hutecitec.

Fritsch, R., Jacobus, A. E., \& Vitelli, R. F. (2020). Diversification, commodification and performance of Brazilian higher education. Revista da Avaliação da Educação Superior, 25(1), 89-112. doi:10.1590/s1414-40772020000100006

Laker, C., Cella, M., Callard, F., \& Wykes, T. (2019). Why is change a challenge in acute mental health wards? A cross-sectional investigation of the relationships between burnout, occupational status and nurses' perceptions of barriers to change. International Journal of Mental Health Nursing, 28(1), 190-198. doi:10.1111/inm. 12517

Larrosa, J. (2011). Experiência e alteridade em educação. Psicologia: Reflexão e Crítica, 19(2), 1-24. doi:10.17058/rea.v19i2.2444

Lei $\mathrm{n}^{\circ}$ 7.498, de 25 de junho de 1986. (1986). Dispõe sobre a regulamentação do exercício da enfermagem e dá outras providências [Internet]. Diário Oficial da República Federativa do Brasil, Brasíli a(DF); 1986. Retrieved in: 8 de agosto de 2021.

Lima, D. W. d. C., Silveira, L. C., Vieira, A. N., Cunha, B. M. C. d., Almeida, A. N. S. d., \& Guerreiro, E. M. (2014). Theoretical references that guide nursing practice in mental health. Escola Anna Nery, 18(2), 336-342. doi:10.5935/1414-8145.20140049

Lira, L. C. S., Silva, P. M. C., Clementino, F. S., \& Barbosa, K. K. S. (2018). Between policies and practices: Therapeutic activities based on harm reduction. Journal of Nursing UFPE, 12(5), 1206-1215. doi:10.5205/1981-8963-v12i5a231307p1206-1215-2018

Lopes, C. S. (2020). How is Brazilian's mental health? The importance of birth cohorts for better understanding the problem. . Cadernos de saude publica, 36(2), e00005020. doi:10.1590/0102-311X00005020

Loyola, C. M. D. (2017). Mental health and psychiatric nursing: Contributions to the resocialization of person in psychic suffering. Escola Anna Nery, 21(3), e20170301. doi:10.1590/2177-9465-ean-2017-0003-0001

Mattos, A., Moll, M., Souto, M., \& Neves, M. (2018). Psychiatric care provided by nursing technicians. Journal of Nursing UFPE, 12(3), 618-625. doi:10.5205/1981-8963-v12i3a25080p618-625-2018

Mesquita, L. F., \& Tavares, C. M. (2020). Systematization of nursing assistance in mental health in primary care: Integrative literature review. Enfermagem Atual in Derme, 91(29). Retrieved from https://revistaenfermagematual.com.br/index.php/revista/article/view/82

Moreira, D. F. N., Santos, I. 1., Azevedo, B. M. E. P., Araujo, D. D., \& Gusmao, R. O. M. (2019). Nursing diagnoses identified in alcohol and other drug users. Enfermagem em Foco, 10(5), 103-108. Retrieved from http://biblioteca.cofen.gov.br/wp-content/uploads/2020/05/Diagn\%C3\%B3sticos-de-enfermagemidentificados-em-usu\%C3\%A1rios-de-\%C3\%A1lcool-e-outras-drogas.pdf

Mota, A., Silva, A., \& Souza, A. (2016). Educação permanente: Práticas e processos com a enfermagem em saúde mental. Revista Portuguesa de Enfermagem de Saúde Mental, 4, 9-16. doi:10.19131/rpesm.0135 
Oliveira, R. C., Silva, L. F., Jesus, M. R., Santos, T. J., Evaristo, T. N., Ribeiro, W. F., . . Gusmão, R. O. M. (2020). Clinical care and the nursing process in mental health: Integrative literature review. Revista Eletrônica Acervo Saúde, 38, e2018. doi:10.25248/reas.e2018.2020

Paes, M. R., Maftum, M. A., \& Mantovani, M. F. (2010). Nursing care to patients with comorbidity clinical and psychiatric in hospital emergency service. Revista Gaucha de Enfermagem, 31(2), 277-284

Pan American Health Organization. (2018). The burden of mental disorders in the region of the Americas. Retrieved from Washington, D.C.: https://iris.paho.org/handle/10665.2/49578

Peplau, H. E. (1962). Interpersonal relations in nursing. New York: Putnam's.

Pessoa Júnior, J. M., Santos, R. C. d. A., Clementino, F. d. S., Nascimento, E. G. C. d., \& Miranda, F. A. N. d. (2016). Mental health education and professional practice in the psychiatric hospital. Texto \& Contexto - Enfermagem, 25(3), e3020015. doi:10.1590/0104-07072016003020015

Rodrigues, J., Santos, S. M. A., \& Spriccigo, J. S. (2012). Teaching nursing care in mental health in undergraduate nursing. Acta Paulista de Enfermagem, 25(6), 844-851. doi:10.1590/S0103-21002012000600004

Salberg, J., Bäckström, J., Röing, M., \& Öster, C. (2019). Ways of understanding nursing in psychiatric patient care - A phenomenographic study. Journal of Nursing Management, 27, 1826-1834. doi:10.1111/jonm.12882

Santos, R. C. A., Pessoa Junior, J. M., \& Miranda, F. A. N. (2018). Psychosocial care network: Adequacy of roles and functions performed by professionals. Revista Gaucha de Enfermagem, 39, e57448. doi:10.1590/1983-1447.2018.57448

Savarimuthu, D. (2020). The potential role of nurses in leading positive behaviour support. British Journal of Nursing, 29(7), 414-418, doi:10.12968/bjon.2020.29.7.414

Serapioni, M. (2019). Franco Basaglia: Biografia de um revolucionário. História, Ciências, Saúde-Manguinhos, 26(4), 1169-1187. doi:10.1590/S010459702019000400008

Sousa, S. S., Costa, L. S. P., \& Jorge, M. S. B. (2019). Cuidado em saúde mental no contexto da atenção primária: Contribuições da enfermagem. Revista Baiana de Saúde Pública, 43(1). doi:10.22278/2318-2660.2019.v43.n1.a3024

Tavares, C. M., Silveira, P. G., Lima, T. O., Ferreira, R. E., \& Santos, G. S. (2015). Changes in undergraduate education in psychiatric nursing and mental health area: Integrative review. Journal of Nursing UFPE, 9(12), 1329-1336. doi:10.5205/reuol.8127-71183-1-SM.0912201535

Travelbee, J. (1969). Intervention-psychiatric nursing: Process in the one-to-one relationship. Philadelphia: Davis.

Vargas, D., Bittencourt, M. N., Rocha, F. M., \& Silva, A. C. O. (2014). Alcohol/drugs community mental health services: Insertion and practices of nursing professionals. Escola Anna Nery, 18(1), 101-106. doi:10.5935/1414-8145.20140015

Vargas, D., Soares, J., Ponce, T. D., \& Oliveira, B. B. (2017). Psychiatric urgency and emergency care nurses: An analysis of their professional and educational profile. Cogitare Enfermagem, 22(4), e50704. doi:10.5380/ce.v22i4.50704 